\title{
Streaming Multimedia via Overlay Networks using Wi-Fi Peer-to-Peer Connections
}

\author{
Poderys, Justas; Soler, José
}

Published in:

Proceedings of 19th IEEE International Symposium on Multimedia

Link to article, DOI:

10.1109/ISM.2017.59

Publication date:

2017

Document Version

Peer reviewed version

Link back to DTU Orbit

Citation (APA):

Poderys, J., \& Soler, J. (2017). Streaming Multimedia via Overlay Networks using Wi-Fi Peer-to-Peer Connections. In Proceedings of 19th IEEE International Symposium on Multimedia IEEE.

https://doi.org/10.1109/ISM.2017.59

\section{General rights}

Copyright and moral rights for the publications made accessible in the public portal are retained by the authors and/or other copyright owners and it is a condition of accessing publications that users recognise and abide by the legal requirements associated with these rights.

- Users may download and print one copy of any publication from the public portal for the purpose of private study or research.

- You may not further distribute the material or use it for any profit-making activity or commercial gain

- You may freely distribute the URL identifying the publication in the public portal

If you believe that this document breaches copyright please contact us providing details, and we will remove access to the work immediately and investigate your claim. 


\title{
Streaming Multimedia via Overlay Networks using Wi-Fi Peer-to-Peer Connections
}

\author{
Justas Poderys and Jose Soler \\ Department of Photonics Engineering \\ Technical University of Denmark \\ Kongens Lyngby, Denmark \\ Email: \{juspo,joss\}@ fotonik.dtu.dk
}

\begin{abstract}
Short range ad-hoc wireless networks can be used to deliver streaming multimedia for information, entertainment and advertisement purposes. To enable short-range communication between various devices, the Wi-Fi Alliance proposed an extension to the IEEE802.11 Wi-Fi standard called Wi-Fi Peer-to-Peer (P2P). It allows compliant devices to form ad-hoc communication groups without interrupting conventional access point-based Wi-Fi communication. This paper proposes to use Wi-Fi P2P connectivity to distribute streaming multimedia in ah-hoc formed user groups. The exchange of multimedia data is performed by forming an overlay network using Peer-to-Peer Streaming Peer Protocol (PPSPP). In order to make PPSPP function over WiFi P2P connections, this paper proposes a number of changes to the protocol. The performance of the proposed system is evaluated using a computer networks emulator. The evaluation is done by streaming Live and Video-on-Demand multimedia among the different size user groups and observing the streaming multimedia Quality-of-Service parameters.
\end{abstract}

\section{INTRODUCTION}

Currently, there is a strong trend of consuming streaming multimedia on personal hand-held devices. The amount of multimedia traffic sent to mobile devices is increasing for several years already, and it is expected to continue to grow by $50 \%$ compounded annual rate between 2016 and 2022 [1]. The adoption of mobile devices for consuming streaming multimedia enables the deployment of new services, such as location-based information, entertainment, and advertising.

At present, the two most widespread short-range wireless communication technologies available in hand-held devices are Bluetooth and Wi-Fi (based on IEEE802.11 standard [2]). While Bluetooth is used predominantly for transmitting low bandwidth data to various connected accessories, Wi-Fi is mainly used for Internet connectivity. However, in conventional infrastructure mode, Wi-Fi users are limited to a single association with an access point (AP), reducing the usability of Wi-Fi for opportunistic ad-hoc networks.

To enable opportunistic short-range ad-hoc communication between devices using Wi-Fi technology, the Wi-Fi Alliance published an extension to the IEEE802.11 standard called WiFi Peer-to-Peer (P2P) [3]. Devices implementing the Wi-Fi $\mathrm{P} 2 \mathrm{P}$ standard can connect to one another without joining a conventional AP-based network. Furthermore, devices using Wi-Fi P2P can maintain connections to an AP and Wi-Fi P2P devices concurrently [3].
Literature in the field already identifies Wi-Fi P2P as a suitable communication technology for ah-hoc opportunistic networks [4]-[6]. However, it was previously shown that delivering streaming multimedia over Wi-Fi P2P connections is not straight-forward. This paper proposes to use an overlay network formed by Peer-to-Peer Streaming Peer Protocol (PPSPP) [7] running over Wi-Fi P2P connections to stream multimedia in an ah-hoc network. The work presented here proposes a set of changes to the PPSPP protocol to make it work over Wi-Fi P2P connections. The proposed changes were verified in small-scale experiments using real Wi-Fi P2P devices. To estimate the performance of multimedia distribution among a larger number of users, an emulated network was used and multimedia streaming parameters, such as playback continuity index and initial buffering time, were analyzed.

\section{RELATED WORK}

A large body of knowledge exists analyzing the Wi-Fi Peerto-Peer (P2P) technology and its specific features. A thorough Wi-Fi P2P technical overview, including experimental evaluation of group formation delays and energy-efficiency, is given in [8]. The authors of [9] analyzed the group owner (GO) selection mechanism in Wi-Fi P2P. In addition to indepth selection mechanism analysis, they propose alternative algorithms for choosing the most capable device as the GO. Additional enhancements to the GO selection mechanism for opportunistic networks are described in [10].

Several papers in the field investigate the possibility of implementing opportunistic networks using Wi-Fi P2P technology in mobile devices. The authors of [4] use experiments with up to three mobile phones to estimate the time required to form concurrent communication groups. The authors of [11] propose to use Wi-Fi P2P to reduce the load on a cellular connection when several devices in close proximity stream the same multimedia content.

In [5] and [6], the authors propose a streaming multimedia delivery system based on Wi-Fi P2P technology. The work presented in [5] describes a fully distributed multimedia streaming system consisting of clients and the master node. The clients act as data caches in the system, while the master node is responsible for connectivity tracking and data exchange scheduling. 


\section{Multimedia Streaming OVER Wi-Fi P2P}

\section{A. Wi-Fi Peer-to-Peer technology}

In conventional wireless networks based on the IEEE802.11 Wi-Fi standard [2], devices operate either in the Infrastructure or Ad-Hoc modes. Devices operating in the infrastructure mode connect to an access point (AP) in order to communicate with each other and potentially other networks. When an AP is not available, two Wi-Fi devices can communicate in an ad-hoc mode by using the reduced functionalities set, called Independent Basic Services Set (IBSS). However, using IBSS lacks most of the functions available in an AP-based communication.

To enable users of Wi-Fi devices to share content and services on their devices on the go, the Wi-Fi Forum adopted an extension to the IEEE802.11 standard called Wi-Fi Peerto-Peer (Wi-Fi P2P, also known as Wi-Fi Direct) [3]. In Wi-Fi $\mathrm{P} 2 \mathrm{P}$, devices communicate by forming groups. In each group, clients implementing the $\mathrm{Wi}-\mathrm{Fi} \mathrm{P} 2 \mathrm{P}$ protocol can have a role of Group Owner (GO) or of a P2P device. Once one of the devices in the group is elected to be the GO, it starts acting as a "soft-AP", performing the functions of an AP.

In contrast to the conventional infrastructure or ad-hoc mode Wi-Fi connections, each device implementing the Wi-Fi P2P standard can act as a GO and be a member of other P2P groups concurrently [8]. From the point of view of software running on the device, each group is exposed as a different virtual network adapter. This implies that Wi-Fi P2P groups can support multi-hop communication (in contrast to ad-hoc connections). If the GO settings support routing of IP packets, then one group member can communicate with another by sending data to the $\mathrm{GO}$, which in turn relays data to the intended member. Furthermore, communication with other networks is possible as well, if the GO supports network address translation.

\section{B. Peer-to-Peer Streaming Peer Protocol}

Multimedia data exchange between the users of the proposed systems was coordinated using the Peer-to-Peer Streaming Peer Protocol (PPSPP) [7]. The choice was motivated by the fact that it is the only protocol for $\mathrm{P} 2 \mathrm{P}$ multimedia streaming standardized by the Internet Engineering Task Force. In the following is a brief description of the main function of the protocol. PPSPP works by dividing multimedia data into a number of fixed-size pieces called chunks. Each chunk is uniquely identified by the chunk number. The work presented here used 1 KByte chunks, following the PPSPP protocol definition.

Once connected, clients periodically exchange information about the chunks they possess by sending HAVE messages. Upon learning that a connected client has chunks not available locally, a requesting client sends REQUEST message containing the chunk numbers to the remote client. Upon receiving the REQUEST message, a client responds by sending the required chunks in DATA messages. The PPSPP protocol does not define the algorithm used to request data chunks. This work used the algorithm that works as follows. The algorithm running in each client maintains a set of all outstanding (already requested, but not received) chunk numbers. Once a second it iterates over the list of connected clients, treating each client equally. If a client has outstanding requests for more than 350 chunks, this client is skipped in this algorithm run. For each remaining client, the algorithm checks if the client has any chunks that are not available locally and not already requested. If such chunk-set exists, the algorithm requests up to the 500th lowest-numbered chunk from the client and then continues processing the next client. The data queries (handling of REQUEST messages) are performed in FIFO order by each client. The above listed threshold values were obtained by experiments.

In addition to exchanging data chunks, the PPSPP protocol defines a procedure to exchange information about other clients in the system. Each client can periodically send PEERREQ messages to all connected clients. Upon receiving PEERREQ message, a client replies with PEERREX message listing the IP addresses and ports of all clients known to it.

\section{Adapting PPSPP for streaming over Wi-Fi P2P}

The functioning of the proposed multimedia streaming system can be divided into data and control planes. The data plane is implemented using Wi-Fi P2P connections and the control plane is implemented using PPSPP protocol. Fig. 1 shows the communication model of the proposed system. Here, each user device operates two virtual Wi-Fi P2P adapters. One adapter is performing the functions of the $\mathrm{GO}$ and acts as a Wi-Fi AP. The second virtual adapter acts as a Wi-Fi P2P client, connecting to the Wi-Fi P2P group hosted by other user (shown as dotted lines in Fig. 1).

The PPSPP protocol, as defined in [7], is meant to be used in IP networks, where all users can establish connections to other users. This is not the case when used with WiFi P2P. Users in a P2P group can communicate only with the GO and possibly other members of the same group. In order for PPSPP to support communication model shown in Fig. 1, several changes were made to the way protocol functions. The protocol was extended to include the MAC address of the client adapter acting as a GO in all connectivity exchange messages. This information allowed PPSPP clients to establish a Wi-Fi P2P connection to other clients and to share information about other clients operating in the system.

Running PPSPP on two virtual adapters has a side effect of sometimes forming two concurrent connections between users over each adapter. In the original PPSPP protocol specification, such situation is solved by checking that all connections are made to clients with unique IP addresses. In the proposed system, each $\mathrm{P} 2 \mathrm{P}$ group has independent IP addresses space and identifying each user based on IP address is not possible. In order to uniquely identify users, each is assigned a type-4 Universally Unique Identifier (UUID) [12]. This UUID value is then added to the HANDSHAKE message that is used to establish a connection between the users. If during a handshake process a user detects that it already has a connection to 


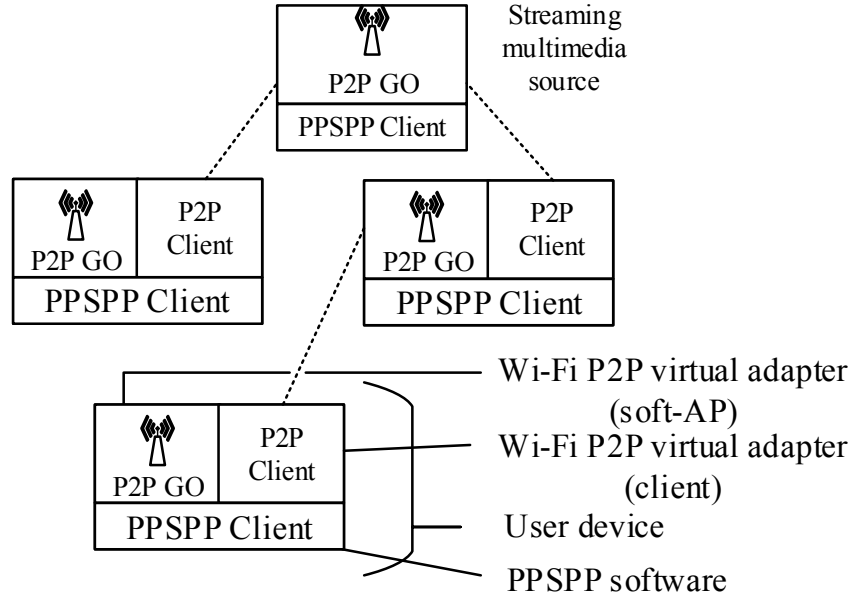

Fig. 1. Communication model of the multimedia streaming system. Each user device performs functions of Wi-Fi P2P GO and P2P client.

another user with the same UUID value, it starts a process to decide which connection should be terminated. To avoid a situation where both users close connections at the same time, a strategy was chosen to decide which user should disconnect: both users compare their UUID values, and the user having a lower UUID value disconnects from a group of a user with the higher value.

\section{PERformance Evaluation}

\section{A. Emulation Model and Experimental Settings}

The basic functions of the system, such as connections establishment and operation over multiple virtual adapters, was verified by running PPSPP client software on Raspberry Pi 3 Model B single-board computers supporting Wi-Fi P2P communication. A large-scale evaluation of the proposed system was performed in a virtual environment using the CORE network emulator [13]. CORE divides the workstation running experiments into a number of virtual instances, each running instance of PPSPP software ${ }^{1}$. The Wi-Fi connections between the clients were emulated using EMANE (part of CORE) and used IEEE 802.11g connections with the maximum data rate set to $54 \mathrm{Mbps}$. All test used a video file having a resolution of 1280x720 px (HD 720p), encoded with a VP8 codec having a bit-rate of 2 Mbps.

In the experiments, two QoS parameters were observed: the Playback Continuity Index (PCI) and the initial buffering time. The PCI is the ratio of the number of rendered video frames in the user's device to the number of attempts to render the next frame. The PCI value can range from 0 (no video frames were rendered in time) to 1 (all frames were rendered in time). The second observed parameter refers to time it takes for the PPSPP client to fill the initial playback buffer and start video playback.

\section{B. Initial Buffering Time}

The average observed client buffering time for both Live and VoD use-cases is shown in Fig. 2a. Note that the tests were carried by increasing the number of users until the PCI value dropped below the threshold value of 0.8 . Fig. $2 \mathrm{~b}$ shows the cumulative distribution function $(\mathrm{CDF})$ of client start-up times in Live, and Fig. 2c in VoD use-cases.

Fig. 2a shows that the size of the buffer has the biggest impact on the start-up time in the Live use-case. This is caused by the fact, that the buffer can only be filled with data that is being produced by the source peer during the filling of the buffer. In the VoD use-case, the whole video file is available for download immediately, and so the initial buffer is filled much faster.

Figures $2 \mathrm{~b}$ and $2 \mathrm{c}$ show how the average start-up times are distributed among the users for different buffer and user group sizes. The CDF for Live use-case shows that when the number of users is large (18), more than half of the users start rendering the multimedia content within the first 3 seconds. In the VoD use-case, all users started rendering multimedia within the first 5 seconds when the number of users was small. However, the distribution is much more long-tailed when the number of users is high.

\section{Playback Continuity}

The average observed playback continuity index (PCI) values for both Live and VoD use-cases are shown in Fig. 3. Fig. 3a shows the average PCI for Live and VoD use-cases based on the initial buffer size. Figures $3 b$ and $3 c$ show the PCI based on the playback buffer size for Live and VoD usecases respectively. In all figures, the horizontal dashed line indicates the threshold PCI value.

Fig. 3a shows that the initial buffer size has a negligible impact on the playback continuity. The PCI stays at 1 (no interruptions to video playback) for as long as the number of users is no larger than 8 in Live use-case and 14 in VoD use case. As the user group size grows, the PCI value gradually decreases to the threshold value for both use-cases.

Fig. $3 b$ indicates that for small user-group sizes (up to 10 users), the size of the playback buffer does not impact the PCI. However, as the user group size increases, having a smaller buffer is more beneficial than having a large one. For a group of 18 users, reducing the buffer size from 2000 data chunks to 500 increases the PCI value from 0.75 to 0.83 .

For the VoD use-case, Fig. 3c shows that for up to 14 users, the size of the buffer has no impact the PCI value. As the number of concurrent users increases, the PCI becomes lower for users using a small buffer. Here it is important to note that users with an unlimited buffer size experienced much lower PCI. Such users requested all multimedia data immediately at the start, increasing the load on the source node. This, in turn, prevented timely delivery of data to the remaining users.

\footnotetext{
${ }^{1}$ Source-code available at github.com/justas-/PyPPSPP
} 


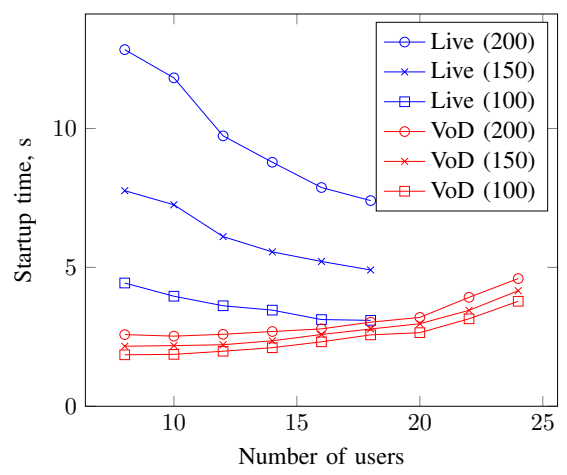

(a) Average VoD and Live start-up time

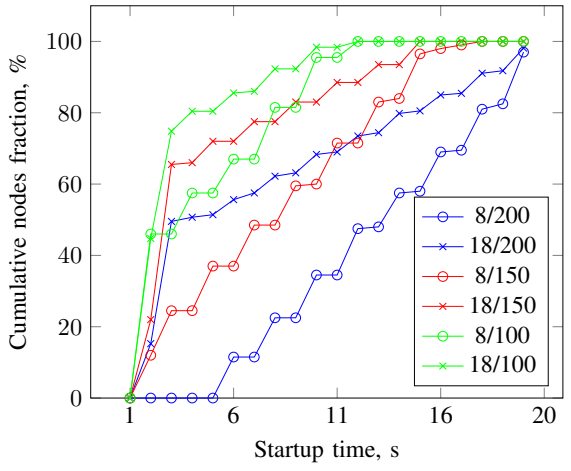

(b) Start-up times distribution (Live)

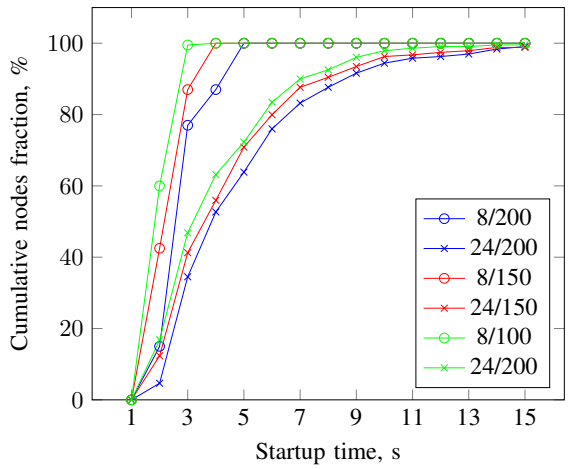

(c) Start-up times distribution $(\mathrm{VoD})$

Fig. 2. Average node start-up time based on multimedia type, initial and playback buffer sizes and number of users. CDF figures have entries in the form of number of users / buffer size.

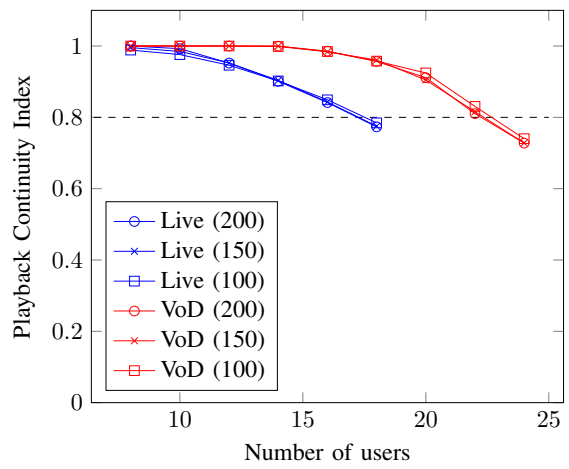

(a) PCI in Live and VoD use-cases

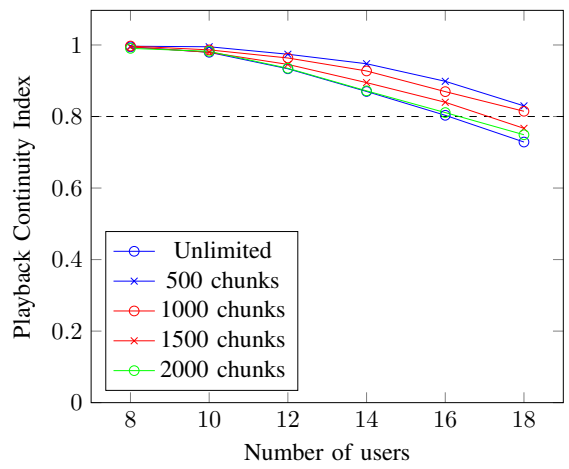

(b) Observed PCI (Live)

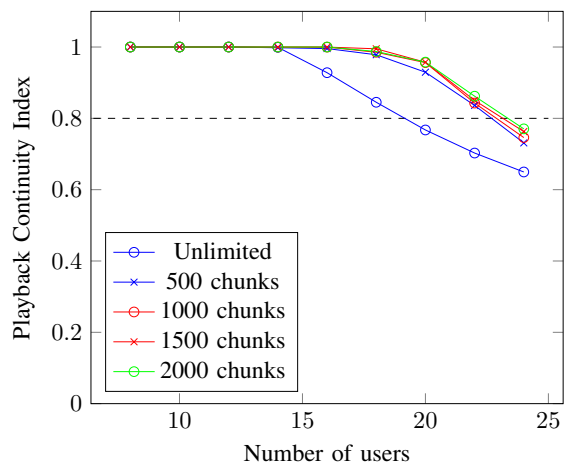

(c) Observed PCI (VoD)

Fig. 3. Observed Playback Continuity Index (PCI) based on multimedia type, initial and playback buffer sizes.

\section{CONCLUSION}

This work presents a $\mathrm{P} 2 \mathrm{P}$ multimedia streaming system operating over the Wi-Fi P2P connections using an extended PPSPP protocol. The experiments using an emulated network indicate that multimedia can be streamed without interruptions to 8 (Live) and 14 (VoD) users. The main obstacle limiting the number of concurrent users in the tested system was lack of coordination between the users during data downloading. This caused longer start-up times and lower multimedia playback continuity. Future work is planned to improve the data delivery coordination between the users and system testing by using different user arrival patterns.

\section{REFERENCES}

[1] "Ericsson mobility report - western europe," Tech. Rep., 2016. [Online]. Available: https://www.ericsson.com/en/mobility-report

[2] "IEEE Standard for Information technology - Telecommunications and information exchange between systems Local and metropolitan area networks. Part 11: Wireless LAN Medium Access Control (MAC) and Physical Layer (PHY) Specifications," Tech. Rep., 2016.

[3] "Wi-Fi Peer-to-Peer (P2P) Technical Specification Version 1.7," Tech Rep., 2016. [Online]. Available: https://www.wi-fi.org/discover-wifi/specifications

[4] M. Conti, F. Delmastro, G. Minutiello, and R. Paris, "Experimenting opportunistic networks with WiFi Direct," in 2013 IFIP Wireless Days (WD). IEEE, nov 2013, pp. 1-6.
[5] H. Yoon and J. Kim, "Collaborative streaming-based media content sharing in wifi-enabled home networks," IEEE Transactions on Consumer Electronics, vol. 56, no. 4, 2010.

[6] H. Yoon, J. Kim, and R. Hsieh, "Peer-assisted video on-demand streaming system in practical wifi-based mobile opportunistic networks," Journal of Network and Computer Applications, vol. 37, pp. 33-44, 2014.

[7] A. Bakker, R. Petrocco, and V. Grishchenko, "Peer-to-Peer Streaming Peer Protocol (PPSPP)," RFC 7574, Jul. 2015.

[8] D. Camps-Mur, A. Garcia-Saavedra, and P. Serrano, "Device-to-device communications with wi-fi direct: overview and experimentation," IEEE wireless communications, vol. 20, no. 3, pp. 96-104, 2013.

[9] M. A. Khan, W. Cherif, and F. Filali, "Group owner election in wi-fi direct," in Ubiquitous Computing, Electronics \& Mobile Communication Conference (UEMCON), IEEE Annual. IEEE, 2016, pp. 1-9.

[10] W. Cherif, M. A. Khan, F. Filali, S. Sharafeddine, and Z. Dawy, "P2p group formation enhancement for opportunistic networks with wi-fi direct," in Wireless Communications and Networking Conference (WCNC), 2017 IEEE. IEEE, 2017, pp. 1-6.

[11] A. Detti, B. Ricci, and N. Blefari-Melazzi, "Mobile peer-to-peer video streaming over information-centric networks," Computer Networks, vol. 81, pp. 272-288, 2015.

[12] P. J. Leach, R. Salz, and M. H. Mealling, "A Universally Unique IDentifier (UUID) URN Namespace,” RFC 4122, Jul. 2005. [Online]. Available: https://rfc-editor.org/rfc/rfc4122.txt

[13] J. Ahrenholz, "Comparison of CORE network emulation platforms," Proceedings - IEEE Military Communications Conference MILCOM, pp. 166-171, 2010. 